\title{
Symmetry breaking in the double moiré superlattices of relaxed twisted bilayer graphene on hexagonal boron nitride
}

\author{
Xianqing $\operatorname{Lin}^{1}{ }^{*}$ and $\mathrm{Jun} \mathrm{Ni}^{2}$ \\ ${ }^{1}$ College of Science, Zhejiang University of Technology, Hangzhou 310023, People's Republic of China \\ ${ }^{2}$ State Key Laboratory of Low-Dimensional Quantum Physics and Frontier Science Center for Quantum Information, \\ Department of Physics, Tsinghua University, Beijing 100084, People's Republic of China
}

(Dated: July 31, 2020)

\begin{abstract}
We study the atomic and electronic structures of the commensurate double moiré superlattices in fully relaxed twisted bilayer graphene (TBG) nearly aligned with the hexagonal boron nitride (BN). The single-particle effective Hamiltonian $\left(\hat{H}^{0}\right)$ taking into account the relaxation effect and the full moiré Hamiltonian introduced by BN has been built for TBG/BN. The mean-field (MF) band structures of the self-consistent Hartree-Fock (SCHF) ground states at different number $(\nu)$ of filled flat bands relative to the charge neutrality point (CNP) are obtained based on $\hat{H}^{0}$ in the plane-wavelike basis. The single-particle flat bands in TBG/BN become separated by the opened gap at CNP due to the symmetry breaking in $\hat{H}^{0}$. We find that the broken $C_{2}$ symmetry in $\hat{H}^{0}$ mainly originates from the intralayer inversion-asymmetric structural deformation in the graphene layer adjacent to BN, which introduces spatially non-uniform modifications of the intralayer Hamiltonian. The gapped flat bands have finite Chern numbers. For TBG/BN with the magic twist angle, the SCHF ground states with $|\nu|=1-3$ are all insulating with narrow MF gaps. When the flat conduction bands are filled, the gap at $\nu=1$ is smaller than that at $\nu=3$, suggesting that the nontrivial topological properties associated with the flat Chern bands are more likely to be observed at $\nu=3$. This is similar for negative $\nu$ with empty valence bands. The dependence of the electronic structure of $\mathrm{TBG} / \mathrm{BN}$ on positive $\nu$ is roughly consistent with recent experimental observations.
\end{abstract}

\section{INTRODUCTION}

The recently realized magic-angle twisted bilayer graphene (TBG) has inspired great interest in exploring its peculiar electronic structure associated with the flat bands around the Fermi level ${ }^{1-5}$. The flat bands in TBG with the magic angle $\left(\theta_{m}\right)$ of about $1.1^{\circ}$ have a total capacity of eight electrons per moiré supercell ${ }^{6-11}$, and the number $(\nu)$ of filled flat bands relative to the charge neutrality point (CNP) can be tuned in the range of $-4 \sim 4$ in experiments ${ }^{1-5}$. The positive and negative $\nu$ are used to represent the number of filled conduction bands and empty valence bands relative to CNP, respectively. Correlated insulating states at integer $\nu$ with $|\nu| \leq 3$ were observed in TBG with $\theta_{m}{ }^{1-5}$. In particular, the quantized anomalous Hall (QAH) effect was realized in TBG aligned with the hexagonal boron nitride $(\mathrm{TBG} / \mathrm{BN})^{12}$. The $\mathrm{QAH}$ state emerges at $\nu=3$ and the insulating state at $\nu=2$ was also observed ${ }^{12}$. Theoretical studies attributed the nontrivial topological properties of TBG/BN to the broken $C_{2}$ symmetry induced by $\mathrm{BN}^{13-16}$. We note that the theoretical models in these studies only included the uniform sublatticeasymmetric potential in the graphene layer on $\mathrm{BN}$, while the nonuniform moiré potentials introduced by $\mathrm{BN}$ have been ignored ${ }^{13-16}$. Moreover, only the rigid TBG or some relaxation effect in TBG with one empirical parameter were considered ${ }^{13-16}$. However, the rigid double moiré superlattices in TBG/BN undergo spontaneous in-plane relaxation and out-of-plane corrugation due to the energy gain from the larger domains of energetically favorable stacking configurations of graphene bilayers and also of graphene on $\mathrm{BN}$, similar to the pristine $\mathrm{TBG}^{17-28}$ and the graphene monolayer on $\mathrm{BN}^{29-32}$. The strong structural deformation in the graphene layer on $\mathrm{BN}$ can play an important role in the symmetry breaking of the single-particle Hamiltonian of the relaxed TBG/BN. The opened gap at CNP in the graphene monolayer aligned with BN has been found to be greatly enhanced by the structural relaxation ${ }^{29-32}$. Therefore, it is important to take into account the full relaxation of TBG/BN and the full effective Hamiltonian introduced by $\mathrm{BN}$ to describe the electronic structure of TBG/BN associated with the broken $C_{2}$ symmetry.

The self-consistent Hartree-Fock (SCHF) method can be used to treat the electron-electron interaction in TBG, while this method was based on the continuum Hamiltonian of TBG with a rigid superlattice or with only the interlayer-spacing corrugation ${ }^{16,33}$. The approach to employing the SCHF method to describe the electronic structure of the fully relaxed TBG/BN remains to be developed.

Here, full relaxation of the commensurate double moiré superlattices in TBG/BN with the twist angle around $\theta_{m}$ is performed and the single-particle effective Hamiltonian $\left(\hat{H}^{0}\right)$ with the relaxation effect and the full moiré Hamiltonian induced by BN taken into account is obtained, based on which the mean-field (MF) band structures of the SCHF ground states are acquired in the plane-wavelike basis. We find that the symmetry breaking in $\hat{H}^{0}$ mainly originates from the broken $C_{2}$ symmetry in the structural deformation of the graphene layer adjacent to $\mathrm{BN}$, which introduces spatially non-uniform modifications of the intralayer Hamiltonian. The gapped flat bands of $\hat{H}^{0}$ have finite Chern numbers. For TBG/BN 


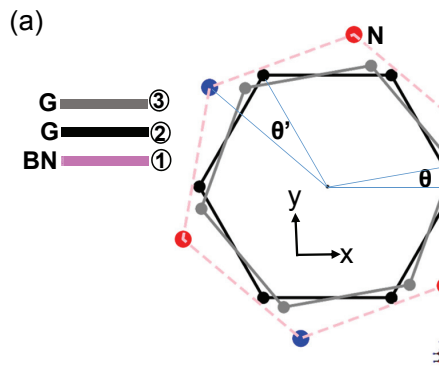

(c)

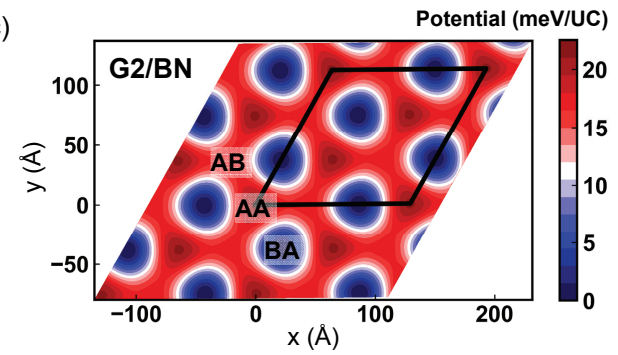

(b)

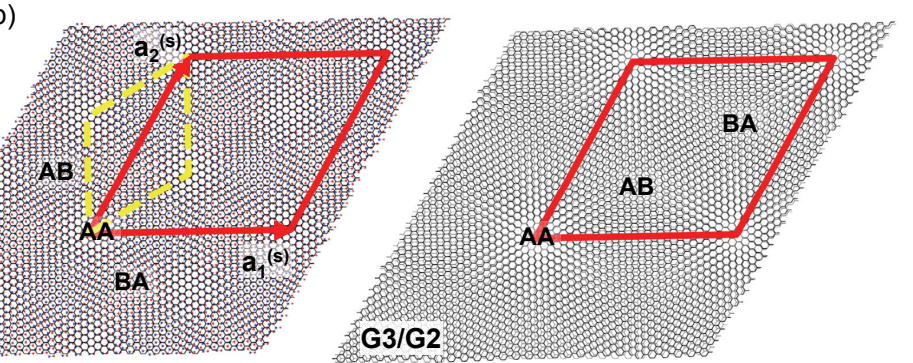

(d)

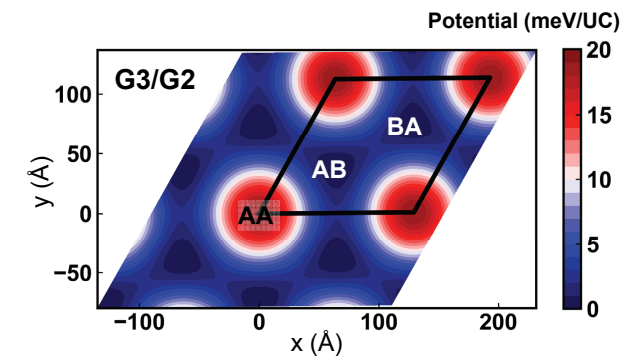

FIG. 1. (Color online) The structure and interlayer interaction potentials of the rigid double moiré superlattices in TBG/BN. (a) The schematic view of the TBG/BN trilayer. The top graphene layer (G3) and the bottom BN layer are rotated by $\theta$ and $\theta^{\prime}$ counterclockwise with respect to the fixed middle graphene layer (G2), respectively. The B and N atoms are represented by blue and red circles, respectively. The lattice-constant mismatch between graphene and BN is amplified in this view and its value is about $-1.70 \%$. (b) The schematic view of the commensurate moiré superlattices in G2 on BN (G2/BN) and in G3 on G2 (G3/G2). $\mathbf{a}_{\mathbf{j}}^{(\mathbf{s})}(j=1,2)$ are the basis vectors of the superlattice in G3/G2 and this superlattice is strictly periodic. An approximate moiré supercell in $\mathrm{G} 2 / \mathrm{BN}$ is indicated by the yellow dashed lines. In parts of G2/BN with BA (AB) stacking, the sublattice-B (sublattice-A) carbon atoms are above the boron (nitrogen) atoms, and the carbon atoms in the other sublattice are above the hexagon centers of $\mathrm{BN}$. (c) and (d) The spatial distribution of the local interlayer interaction potential as a function of the position in the rigid superlattices of G2/BN (c) and G3/G2 (d) for commensurate TBG/BN with $\theta=1.08^{\circ}$. The potential is in units of meV per graphene unit cell (UC).

with $\theta_{m}$, the SCHF ground states with $|\nu|=1-3$ are all insulating with narrow MF gaps.

The outline of this paper is as follows: In Sec. II we present the geometry and the structural relaxation of the double moiré superlattices in TBG/BN. For the relaxed TBG/BN, the symmetry breaking in $\hat{H}^{0}$ is studied in Sec. III and the MF electronic structure at integer band filling is shown in Sec. IV. Section V presents the summary and conclusions.

\section{RELAXATION OF THE DOUBLE MOIRÉ SUPERLATTICES}

Placing TBG on a BN monolayer with their orientations nearly aligned, the double moiré superlattices emerge in the TBG/BN trilayer, as depicted in Figs. 1(a) and $1(\mathrm{~b})$. We consider the TBG/BN with the twist angle $(\theta)$ between the top graphene layer (G3) and the fixed middle graphene layer $(\mathrm{G} 2)$ close to $\theta_{m}$.

The spanning vectors of the moiré superlattice in G3 on G2 (G3/G2) are along the armchair directions of graphene, while those of the moiré superlattice in G2 on $\mathrm{BN}(\mathrm{G} 2 / \mathrm{BN})$ with perfect alignment are along the zigzag directions. The lattice-constant mismatch between G2 and $\mathrm{BN}$ and also the relative twist give rise to the moiré superlattice in G2/BN. The calculated lattice constant of graphene $(a)$ is $2.447 \AA$ and that of the BN monolayer $\left(a^{\prime}\right)$ is $2.489 \AA$, where $a=(1+\epsilon) a^{\prime}$ with $\epsilon=-1.70 \%$. Since the double moiré superlattices have similar sizes but distinct orientations when TBG with $\theta_{m}$ is perfectly aligned with BN, these superlattices are incommensurate. To obtain the commensurate double superlattices, the BN layer has to be rotated by a small angle $\left(\theta^{\prime}\right)$ with respect to the fixed G2 layer. We find that when $\theta^{\prime}$ is around $1.6^{\circ}$ and the lattice-constant mismatch between graphene and BN is maintained around $-1.70 \%$, the superlattices become commensurate and can be strictly periodic. The size of the moiré supercell in $\mathrm{G} 2 / \mathrm{BN}$ is just a third of that in G3/G2, as indicated in Fig. 1(b). Such strictly commensurate double moiré superlattices with tuned geometry parameters allow us to theoretically study the electronic structure of fully relaxed TBG/BN in the plane-wave-like basis. The geometry of the double moiré superlattices in TBG/BN is detailed in the Appendix and the geometry parameters of the considered commensurate TBG/BN are listed in Table I.

The rigid moiré superlattices undergo spontaneous inplane relaxation due to the energy gain from the larger domains of energetically favorable stacking configurations. Each layer is also corrugated to reach the optimal interlayer distances of the varying stacking con- 

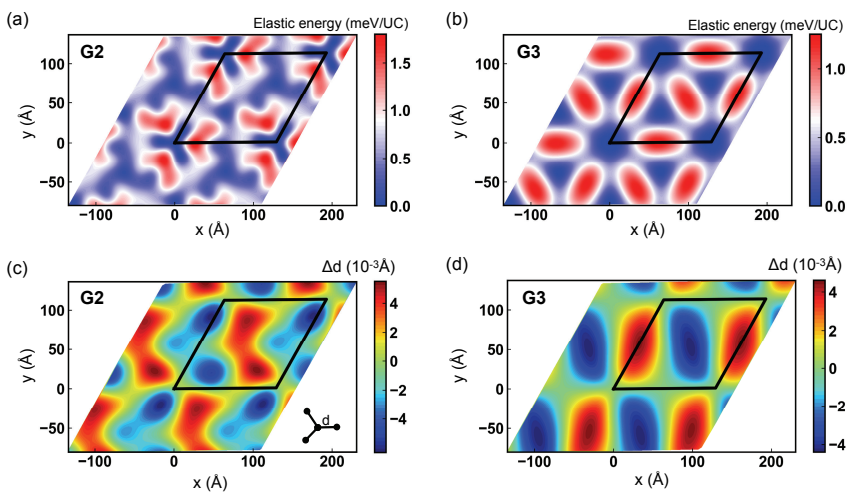

FIG. 2. (Color online) The in-plane structural deformation in the graphene layers of the relaxed TBG/BN with $\theta=1.08^{\circ}$. (a) and (b) The spatial distribution of the local elastic energy in G2 (a) and G3 (b). (c) and (d) The distribution of $\Delta d=$ $d-d_{0}$ in G2 (c) and G3 (d), where $d$ is the length of a C$\mathrm{C}$ bond along the $x$ direction (see the inset) and $d_{0}$ is the bond length of the rigid graphene. An periodic supercell is indicated by the black lines in (a-d).

figurations across the superlattices. In the superlattice between graphene layers, the interlayer interaction potential $(V)$ has the lowest and the same value at the ABand BA-stacked parts, and its spatial variation is inversion symmetric with respect to the AA-stacked part at the origin, as shown in Fig. 1(d). The symmetry of $V$ is due to the $C_{2}$ symmetry in the rigid structure of TBG with respect to the vertical axis for the relative rotation of the graphene layers. Within each graphene layer, $\mathrm{C}_{2}$ becomes the inversion symmetry with respect to the hexagon center at the origin. Upon relaxation in the pristine TBG, the regions with AB-like and BA-like stackings increase in size and the $C_{2}$ symmetry is maintained. In contrast, the $C_{2}$ symmetry is absent in the BN layer and also in the superlattice of $\mathrm{G} 2 / \mathrm{BN}$. The BA-stacked parts in $\mathrm{G} 2 / \mathrm{BN}$ have a much lower interlayer interaction potential $\left(V^{\prime}\right)$ than that at the AB-stacked parts, leading to an inversion-asymmetric distribution of $V^{\prime}$, as shown in Fig. 1(c). Then, only the regions with BA-like stackings tend to become larger upon relaxation in $\mathrm{G} 2 / \mathrm{BN}$. Therefore, subject to both $V$ and $V^{\prime}$, the $C_{2}$ symmetry in G2 layer is broken upon relaxation, and the in-plane structural deformation in G2 becomes distinct from that in the relaxed pristine TBG. We have performed the full relaxation of $\mathrm{TBG} / \mathrm{BN}$ employing the continuum elastic theory, as detailed in the Appendix.

To demonstrate the broken symmetry in G2 of the relaxed TBG/BN and to compare the in-plane structural deformation in G2 and G3, the spatial distributions of the elastic energy induced by the strain field in the graphene layers are illustrated in Figs. 2(a) and 2(b). Since the relaxation of G3 is driven by the interaction between graphene layers, the spatial variation of the elastic energy in G3 is similar to that in the pristine TBG. The regions with high elastic energy in G3 are located between the AB- and BA-stacked parts, and the distribution is ap-
TABLE I. The geometry parameters and electronic properties of the studied commensurate double moiré superlattices in TBG/BN: $N$ used to express the supercell basis vectors, the twist angle $(\theta)$ between G3 and G2, the lattice-constant mismatch between graphene and $\mathrm{BN}(\epsilon)$, the twist angle $\left(\theta^{\prime}\right)$ between $\mathrm{G} 2$ and $\mathrm{BN}$, the widthes of the single-particle flat valence $\left(W_{v}\right)$ and conduction $\left(W_{c}\right)$ bands, the single-particle gap $\left(\Delta_{0}\right)$ at $\mathrm{CNP}$, and the $\mathrm{MF}$ gaps $\left(\Delta_{\nu}^{\mathrm{MF}}\right)$ in the SCHF ground states at different number $(\nu)$ of filled flat bands. $W_{v}$, $W_{c}, \Delta_{0}$, and $\Delta_{\nu}^{\mathrm{MF}}$ are in units of $\mathrm{meV}$.

\begin{tabular}{rrrr}
\hline \hline$N$ & 30 & 29 & 28 \\
\hline$\theta$ & $1.0845^{\circ}$ & $1.1213^{\circ}$ & $1.1607^{\circ}$ \\
$\epsilon$ & $-1.6255 \%$ & $-1.6801 \%$ & $-1.7385 \%$ \\
$\theta^{\prime}$ & $1.6448^{\circ}$ & $1.7012^{\circ}$ & $1.7616^{\circ}$ \\
$W_{v}$ & 2.75 & 3.70 & 7.83 \\
$W_{c}$ & 2.50 & 7.23 & 12.27 \\
$\Delta_{0}$ & 6.92 & 7.85 & 8.64 \\
$\Delta_{1}^{\mathrm{MF}}$ & 3.76 & 13.41 & 19.49 \\
$\Delta_{2}^{\mathrm{MF}}$ & 3.51 & 15.08 & 20.48 \\
$\Delta_{3}^{\mathrm{MF}}$ & 4.78 & 16.74 & 21.17 \\
$\Delta_{-1}^{\mathrm{MF}}$ & 5.76 & 10.36 & 17.73 \\
$\Delta_{-2}^{\mathrm{MF}}$ & 6.92 & 11.01 & 18.80 \\
$\Delta_{-3}^{\mathrm{MF}}$ & 7.63 & 12.59 & 17.12 \\
\hline \hline
\end{tabular}

proximately inversion symmetric. In contrast, the inversion symmetry is absent in the elastic-energy distribution of G2. The regions in G2 between parts with BA stacking of $\mathrm{G} 2 / \mathrm{BN}$ have higher elastic energy than the positions between parts with $\mathrm{AB}$ and BA stackings of G3/G2. In addition, the highest elastic energy in G2 is much larger than that in G3, indicating stronger relaxation in G2.

The elastic-energy distribution reflects the spatial variation of the strain field in each graphene layer. As we will show in the next section, the strain field induces changes in the intralayer on-site energies and hopping terms of the Hamiltonian. The changing of the intralayer hopping terms is due to the modified $\mathrm{C}-\mathrm{C}$ bond lengthes $(d)$ by strain. Figures $2(\mathrm{c})$ and $2(\mathrm{~d})$ show the spatial variation of $\Delta d=d-d_{0}$ for the $\mathrm{C}-\mathrm{C}$ bonds along the $x$ direction in G2 and G3, respectively, where $d_{0}$ is the bond length of the rigid graphene. The bonds can be stretched or shortened by strain. The regions with large $|\Delta d|$ in G2 are distinct from those in G3, and the highest $|\Delta d|$ in G2 is larger than that in G3. In particular, the distribution of $|\Delta d|$ in G2 has no inversion symmetry in contrast to that in G3.

\section{SYMMETRY BREAKING IN THE SINGLE-PARTICLE HAMILTONIAN OF RELAXED TBG/BN}

For the relaxed TBG/BN, we have built an effective single-particle Hamiltonian $\hat{H}^{0}$ for the moiré superlattice in G3/G2 by extending the Hamiltonian of $p_{z}$ orbitals for graphene bilayers proposed in Refs. [25, 34] and the effective Hamiltonian of monolayer graphene on BN proposed 
in Ref. [32]. This effective Hamiltonian reads

$$
\begin{aligned}
\hat{H}^{0} & =\sum_{n=2}^{3} \sum_{i} \varepsilon_{n, i} c_{n, i}^{\dagger} c_{n, i}+\sum_{n=2}^{3} \sum_{\langle i, j\rangle} t_{i, j}^{(n, n)}\left(c_{n, i}^{\dagger} c_{n, j}+\text { h.c. }\right) \\
& +\sum_{i, j} t_{i, j}^{(2,3)}\left(c_{2, i}^{\dagger} c_{3, j}+\text { h.c. }\right),
\end{aligned}
$$

where $c_{n, i}^{\dagger}(n=2,3)$ is the creation and $c_{n, i}$ is the annihilation operator of a $p_{z}$-like orbital at the site $i$ in the $\mathrm{G} n$ layer, $\langle i, j\rangle$ denotes the intralayer nearest neighbors, and the on-site energies, intralayer and interlayer hopping terms are represented by $\varepsilon_{n, i}, t_{i, j}^{(n, n)}$, and $t_{i, j}^{(2,3)}$, respectively. The used parameters to obtain these onsite and hopping terms are extracted from the ab-initio electronic structures of shifted bilayers.

For the intralayer on-site and hopping terms, we take into account the effect of BN and the in-plane strain. The strain modifies the bond lengthes in graphene and thus influences the intralayer hopping terms. The hopping $t_{i, j}^{(n, n)}$ between intralayer nearest neighbors with their distances $d$ deviated from that of the pristine graphene $d_{0}=a / \sqrt{3}$ is given by

$$
V_{p p \pi}(d, \boldsymbol{r})=-V_{p p \pi}^{0}(\boldsymbol{r}) e^{-\left(d-d_{0}\right) / \lambda_{\pi}}
$$

with $\lambda_{\pi}=0.47 \AA$. The $V_{p p \pi}^{0}$ for G3 is a constant and is taken to be $2.598 \mathrm{eV}$, while the $V_{p p \pi}^{0}$ for G2 depends on the local shift vector $\boldsymbol{\delta}^{\prime}(\boldsymbol{r})$ between G2 and BN with $V_{p p \pi}^{0}\left(\boldsymbol{\delta}^{\prime}\right)$ given by the effective model in Ref. [32]. In G2, the on-site energies in the two sublattices $\left(\varepsilon_{A}\right.$ and $\left.\varepsilon_{B}\right)$ also vary with $\boldsymbol{\delta}^{\prime}$ and can be computed by the model in Ref. [32]. Moreover, the in-plane strain induces changes in $\varepsilon_{m}(m=\mathrm{A}, \mathrm{B})$ for both G2 and G3 expressed as

$$
\delta \varepsilon_{m}=\alpha_{0}\left(\frac{\partial u_{x}^{(n)}}{\partial x}+\frac{\partial u_{y}^{(n)}}{\partial y}\right)
$$

where $n=2,3$, and $\alpha_{0}=-4.95 \mathrm{eV}$. Calculations show that the $\delta \varepsilon_{i}$ in G3 is smaller than $1 \mathrm{meV}$, while it can be rather large in $\mathrm{G} 2$.

The interlayer hopping $t_{i, j}^{(2,3)}$ between sites in G2 and G3 with in-plane projection $r$ and out-of-plane projection $h$ is expressed as

$$
V_{p p \sigma}(r, h)=V_{p p \sigma}^{0} e^{-\left(h-h_{0}\right) / \lambda^{\prime}} e^{-\left(\sqrt{r^{2}+h^{2}}-h\right) / \lambda} \frac{h^{2}}{r^{2}+h^{2}},
$$

where $V_{p p \sigma}^{0}=0.381 \mathrm{eV}, h_{0}=3.32 \AA, \lambda^{\prime}=0.58 \AA$, and $\lambda$ $=0.27 \AA$. All interlayer hopping terms with $r \leq 5.0 \AA$ are included in the calculations. The local optimal interlayer spacing obtained from ab-initio calculations is given by $h(\boldsymbol{\delta})=3.413+0.0622 \sum_{j=1}^{3} \cos \left(\mathbf{G}_{j} \cdot \boldsymbol{\delta}\right)$ in units of $\AA$. We note that these Hamiltonian parameters can reproduce the observed $\theta_{m}$ of the pristine TBG.

Since the moiré supercell in G3/G2 is rather large, the Hamiltonian $\hat{H}^{0}$ can be expressed using the plane-wavelike basis functions. In this approach, the atomic positions of the rigid graphene lattice in each layer are used to label the hopping sites in the Hamiltonian. The planewave-like basis functions are labeled with the valley index $(\xi= \pm)$, the sublattice and layer index $(\alpha=\mathrm{A} 2, \mathrm{~B} 2, \mathrm{~A} 3$, B3), a k-point $\left(\mathbf{k}^{(s)}\right)$ in the supercell BZ, and a reciprocal lattice vector $\left(\mathbf{G}^{(\mathbf{s})}\right)$ of the superlattice. They are defined as

$$
\begin{aligned}
\mid \alpha, \mathbf{k}^{(s)}+ & \left.\mathbf{k}_{\xi}+\mathbf{G}^{(s)}\right\rangle= \\
& \frac{1}{\sqrt{\tilde{N}}} \sum_{\mathbf{r}_{\alpha}} e^{i\left(\mathbf{k}^{(s)}+\mathbf{k}_{\xi}+\mathbf{G}^{(s)}\right) \cdot \mathbf{r}_{\alpha}}\left|\mathbf{r}_{\alpha}\right\rangle,
\end{aligned}
$$

where $\mathbf{k}_{\xi}$ is the center of one of the supercell BZs containing the Dirac points of each layer at their corners in the $\xi$ valley and $\mathbf{r}_{\alpha}$ is the rigid in-plane position of a site in the sublattice $\alpha$ of the corresponding layer. $\mathbf{k}_{\xi}$ is thus a reciprocal lattice vector of the supercell and the used $\mathbf{k}_{\xi}$ can be seen in the schematic reciprocal lattice of a moiré superlattice in Fig. 7(a) of the Appendix. We use the 37 shortest $\mathbf{G}^{(\mathbf{s})}$ shown in Fig. 7(b). The $\hat{H}^{0}$ element between two basis functions is given by

$$
\begin{array}{r}
H_{\alpha, \mathbf{G}^{(s)^{\prime}} ; \beta, \mathbf{G}^{(s)}}^{0}\left(\xi, \mathbf{k}^{(s)}\right)=\left\langle\alpha, \mathbf{k}^{\prime}\left|\hat{H}^{0}\right| \beta, \mathbf{k}\right\rangle= \\
\quad \frac{1}{\tilde{N}_{0}} \sum_{\mathbf{r}_{\alpha} \in S C} \sum_{\mathbf{r}_{\beta}} e^{-i \mathbf{k}^{\prime} \cdot \mathbf{r}_{\alpha}+i \mathbf{k} \cdot \mathbf{r}_{\beta}}\left\langle\mathbf{r}_{\alpha}\left|\hat{H}^{0}\right| \mathbf{r}_{\beta}\right\rangle,
\end{array}
$$

where $\mathbf{k}^{\prime}=\mathbf{k}^{(s)}+\mathbf{k}_{\xi}+\mathbf{G}^{(s)^{\prime}}, \mathbf{k}=\mathbf{k}^{(s)}+\mathbf{k}_{\xi}+\mathbf{G}^{(s)}$, the summation over $\mathbf{r}_{\alpha}$ is done in a supercell, and $\tilde{N}_{0}$ is the number of graphene unit cells in one layer of the supercell. $\left\langle\mathbf{r}_{\alpha}\left|\hat{H}^{0}\right| \mathbf{r}_{\beta}\right\rangle$ represents the on-site and hopping terms given above. For each $\mathbf{r}_{\alpha}$, only a small number of large $\left\langle\mathbf{r}_{\alpha}\left|\hat{H}^{0}\right| \mathbf{r}_{\beta}\right\rangle$ are required in the summation of Eq. (6). Since the Hamiltonian between states from two different valleys is negligible for large moiré superlattices, the bands from $H^{0}$ are valley polarized. The Berry curvature of the band states and the Chern number of each band can be calculated for the Hamiltonian in Eq. (6) using the method in Ref. [34].

The single-particle band structure of the relaxed TBG/BN with $\theta=1.08^{\circ}$ is displayed in Fig. 3(a). In contrast to the pristine TBG with degenerate conduction and valence band states at $K^{(s)}$, the flat bands of TBG/BN are separated by the opened gap. This is due to the broken $C_{2}$ symmetry in $\hat{H}^{0}$ by both relaxation and BN. For TBG/BN with $\theta=1.08^{\circ}$, the gap $\left(\Delta_{0}\right)$ at $\mathrm{CNP}$ reaches $6.92 \mathrm{meV}$. The flat conduction and valence bands in TBG/BN have similar band widthes of about $2.5 \mathrm{meV}$, while the flat valence bands in the pristine TBG are much narrower than the flat conduction bands. In both TBG/BN and the pristine TBG, the flat bands are well gapped from other dispersive bands. Since the flat valence and conduction bands have become separated in TBG/BN, the Chern number $\left(C_{n}\right)$ of each flat band can be computed to characterize its topological property. In the $\xi=+$ valley, the flat valence and conduction bands have finite $C_{n}$ of +1 and -1 , respectively. The $C_{n}$ of each flat band in the $\xi=-$ valley is just the opposite of that for $\xi=+$, as labeled in Fig. 3(a). The finite $C_{n}$ of the flat bands are contributed by the large Berry 
(a)

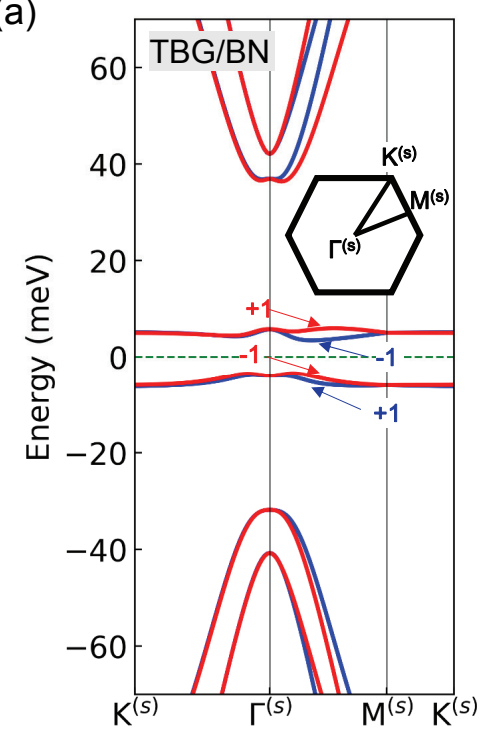

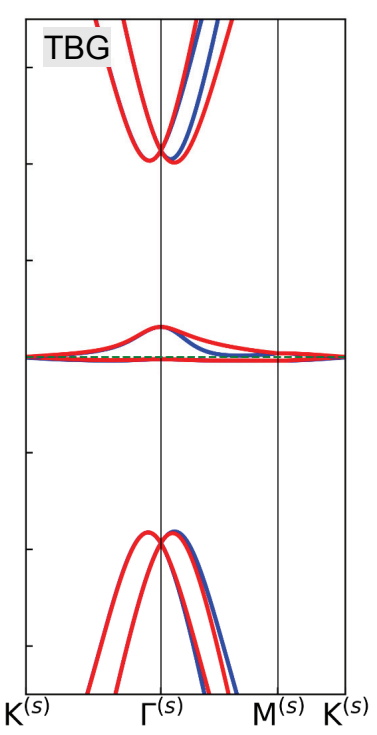

(b)
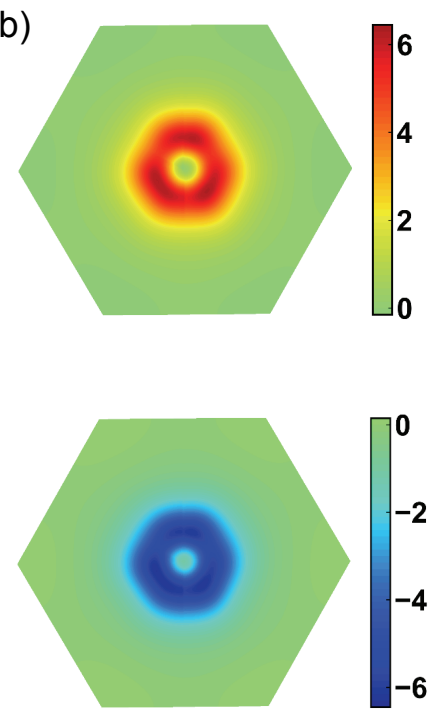

FIG. 3. (Color online) (a) The single-particle band structures of the relaxed TBG/BN and pristine TBG with $\theta=1.08^{\circ}$. The blue and red lines represent bands in the $\xi=+$ and $\xi=-$ valleys, respectively. The Fermi levels are set to be zero and are represented by the dashed green lines. The Chern number of each flat band is labeled. (b) The distribution of the Berry curvature in units of $\AA^{2}$ in the supercell BZ for the flat valence (top panel) and conduction (bottom panel) bands in the $\xi=+$ valley.

curvature at k-points around the center of the supercell Brillouin zone (BZ), as shown in Fig. 3(b).

The main characteristics of the electronic structure of relaxed $\mathrm{TBG} / \mathrm{BN}$ in contrast to the pristine $\mathrm{TBG}$ are caused by the broken $C_{2}$ symmetry in $\hat{H}^{0}$. We will reveal the origin of this symmetry breaking in the following. The BN layer introduces an effective Hamiltonian in G2, whose on-site and hopping terms lack the $C_{2}$ symmetry. The $C_{2}$ symmetry is present in the atomic structure of the pristine TBG, while it is broken in the relaxed structure of G3/G2 in TBG/BN. The structural deformation without the $C_{2}$ symmetry also induces changes in the intralayer on-site and hopping terms as well as the interlayer hopping terms compared with those of the pristine TBG. In Fig. 4(a), the single-particle band structure of the relaxed TBG/BN is compared with that of G3/G2 ignoring the effective Hamiltonian in G2 introduced by BN while still considering the interlayer potential between G2 and $\mathrm{BN}$ for the structural relaxation. We find that the flat bands have similar dispersions, and the gap $\Delta_{0}$ is just reduced by $1.3 \mathrm{meV}$ when the electronic contribution of $\mathrm{BN}$ is ignored. This indicates that the symmetry breaking in $H^{0}$ mainly originates from the broken $C_{2}$ symmetry in the relaxed structure of G3/G2 in TBG/BN.

For the pristine TBG, $\hat{H}^{0}$ remains the same under the operation of $C_{2}$, that is $\hat{H}^{0}=C_{2} \hat{H}^{0} C_{2}$. In addition, the $C_{2}$ operation transforms the plane-wave-like basis functions defined in Eq. (5) as $C_{2}|\tilde{\alpha} n, \mathbf{k}\rangle=|(-\tilde{\alpha}) n,-\mathbf{k}\rangle$, where $n$ is the layer index and $\tilde{\alpha}=+$ and $\tilde{\alpha}=-$ denote the $\mathrm{A}$ and $\mathrm{B}$ sublattices, respectively. Considering both the $C_{2}$ and time reversal $(\mathcal{T})$ symme- tries, the elements of $\hat{H}^{0}$ for the pristine TBG satisfy $\left\langle\tilde{\alpha}^{\prime} n^{\prime}, \mathbf{k}^{\prime}\left|\hat{H}^{0}\right| \tilde{\alpha} n, \mathbf{k}\right\rangle=\left\langle\left(-\tilde{\alpha}^{\prime}\right) n^{\prime}, \mathbf{k}^{\prime}\left|\hat{H}^{0}\right|(-\tilde{\alpha}) n, \mathbf{k}\right\rangle^{*}$. Then, the magnitude of the difference $\mid\left\langle\tilde{\alpha}^{\prime} n^{\prime}, \mathbf{k}^{\prime}\left|\hat{H}^{0}\right| \tilde{\alpha} n, \mathbf{k}\right\rangle-$ $\left\langle\left(-\tilde{\alpha}^{\prime}\right) n^{\prime}, \mathbf{k}^{\prime}\left|\hat{H}^{0}\right|(-\tilde{\alpha}) n, \mathbf{k}\right\rangle^{*} \mid$ can be used to indicate the strength of $\hat{H}^{0}$ terms associated with the broken $C_{2}$ symmetry in TBG/BN.

We take the $H_{0}$ at $K^{(s)}$ in the $\xi=+$ valley as an example and denote $\left\langle\tilde{\alpha}^{\prime} n^{\prime}, \mathbf{k}^{\prime}\left|H^{0}\right| \tilde{\alpha} n, \mathbf{k}\right\rangle$ with $\mathbf{k}^{\prime}=K^{(s)}+\mathbf{k}_{+}+\mathbf{G}^{(s)^{\prime}}$ and $\mathbf{k}=K^{(s)}+\mathbf{k}_{+}+$ $\mathbf{G}^{(s)}$ by $H_{\tilde{\alpha}^{\prime} n^{\prime}, \mathbf{G}^{(s)^{\prime}} ; \tilde{\alpha} n, \mathbf{G}^{(s)}}^{0}$. The elements $H_{\tilde{\alpha}^{\prime} n^{\prime}, \mathbf{0} ; \tilde{\alpha} n, \mathbf{G}^{(s)}}^{0}$ with $\mathbf{G}^{\left(s^{\prime}\right.}=\mathbf{0}$ are considered. Their correspond-

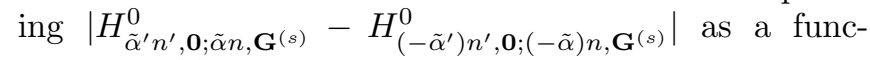
tion of $\mathbf{G}^{(s)}$ are illustrated in Figs. 4(b)-4(d). The $H_{A 2, \mathbf{0} ; A 2, \mathbf{G}^{(s)}}^{0}$ and $H_{B 2, \mathbf{0} ; B 2, \mathbf{G}^{(s)}}^{0}$ are contributed by the on-site energies $\varepsilon_{A}$ and $\varepsilon_{B}$ in G2, which are mainly induced by the strain field [see Eq. (3)]. We find that $\left|H_{A 2, \mathbf{0} ; A 2, \mathbf{G}^{(s)}}^{0}-H_{B 2, \mathbf{0} ; B 2, \mathbf{G}^{(s)}}^{0 *}\right|$ has large values only at the shortest nonzero reciprocal lattice vectors of the moiré superlattice in G2/BN, and the value at zero $\mathbf{G}^{(s)}$ is finite but small. This implies that the symmetry breaking in the on-site terms of $\hat{H}^{0}$ is mainly due to the nonuniform inversion-asymmetric spatial variation of $\varepsilon_{A}$ and $\varepsilon_{B}$ in G2 [see Fig. 4(e)] rather than the uniform $\varepsilon_{A}-\varepsilon_{B}$. Such distribution of on-site energies in G2 is just due to the absence of inversion symmetry in the strain field shown in Fig. 2(a). In contrast, the on-site energies in G3 are approximately symmetric but are all smaller than $1 \mathrm{meV}$.

The intralayer hopping between nearest neighbors changes when the bond lengthes $d$ deviate from $d_{0}$ of the pristine graphene [see Eq. (2)]. In G3, the distribution 
(a)

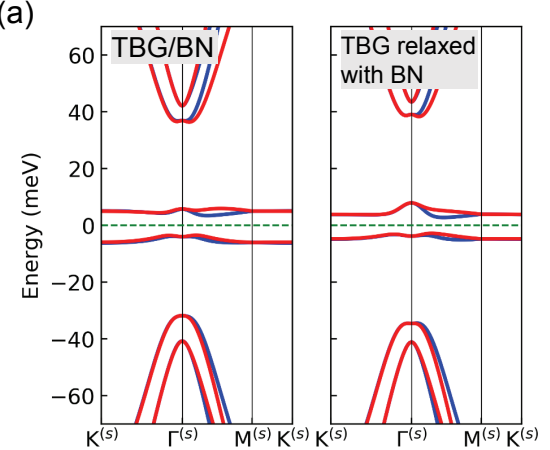

(d)

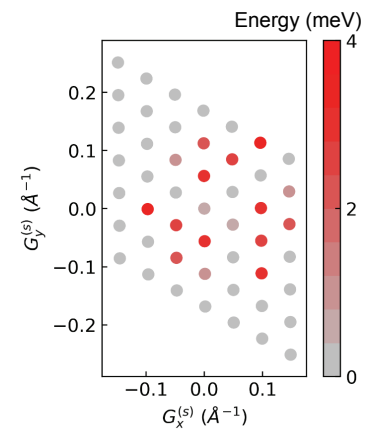

(b)

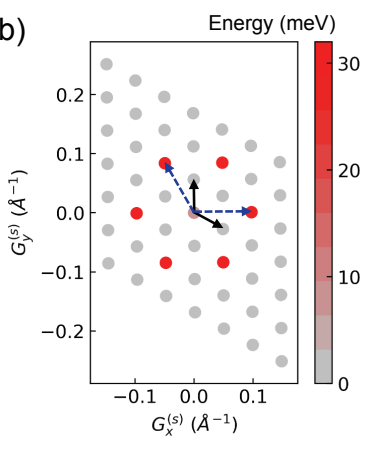

(c)

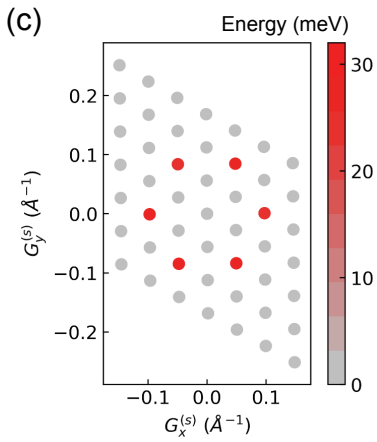

(e)

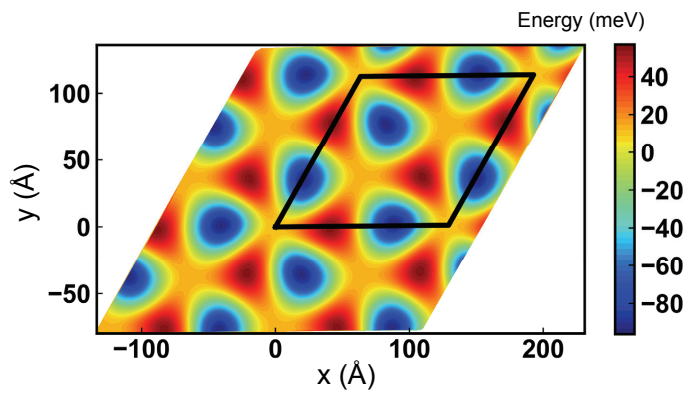

FIG. 4. (Color online) (a) The single-particle band structure of the relaxed TBG/BN with $\theta=1.08^{\circ}$ compared with that of G3/G2 ignoring the effective Hamiltonian in G2 introduced by BN while still considering the interlayer potential between G2 and $\mathrm{BN}$ for the structural relaxation. (b)-(d) The differences between the $H^{0}$ elements due to the broken $C_{2}$ symmetry as a function of $\mathbf{G}^{(s)}$ for the $\xi=+$ valley and the $K^{(s)}$ point: $\left|H_{A 2, \mathbf{0} ; A 2, \mathbf{G}^{(s)}}^{0}-H_{B 2, \mathbf{0} ; B 2, \mathbf{G}^{(s)}}^{0 *}\right|(\mathrm{b}),\left|H_{A 2, \mathbf{0} ; B 2, \mathbf{G}^{(s)}}^{0}-H_{B 2, \mathbf{0} ; A 2, \mathbf{G}^{(s)}}^{0 *}\right|$ (c), and $\mid H_{A 2, \mathbf{0} ; A 3, \mathbf{G}^{(s)}}^{0}-H_{B 2, \mathbf{0} ; B 3, \mathbf{G}^{(s)}}^{0 *}$ (d). In (b) the solid and dashed lines represent the spanning vectors of the reciprocal lattices of the moiré superlattices in G3/G2 and G2/BN, respectively. (e) The distribution of the average on-site energies $\left(\varepsilon_{A}+\varepsilon_{B}\right) / 2$ in $\mathrm{G} 2$.

of $\Delta d=d-d_{0}$ is approximately inversion symmetric, as seen in Fig. 2(c), the symmetry is thus maintained for the hopping terms in G3. However, the inversionasymmetric $\Delta d$ in G2 [see Fig. 2(d)] leads to large $\left|H_{A 2, \mathbf{0} ; B 2, \mathbf{G}^{(s)}}^{0}-H_{B 2, \mathbf{0} ; A 2, \mathbf{G}^{(s)}}^{0 *}\right|$, which is contributed by the intralayer hopping in G2, as shown in Fig. 4(c). The locations with high values in Fig. 4(c) are the same as those in Fig. 4(b) and the magnitudes in both figures are similar. The symmetry of the interlayer hopping is also affected by the structural deformation in G2, while the influence is much weaker, as indicated by the small $\left|H_{A 2, \mathbf{0} ; A 3, \mathbf{G}^{(s)}}^{0}-H_{B 2, \mathbf{0} ; B 3, \mathbf{G}^{(s)}}^{0 *}\right|$ shown in Fig. $4(\mathrm{~d})$.

\section{MEAN-FIELD BAND STRUCTURES AT INTEGER BAND FILLING}

When an integer number more or less flat bands are filled relative to $\mathrm{CNP}$ in relaxed $\mathrm{TBG} / \mathrm{BN}$, electronelectron (e-e) interaction has to be taken into account explicitly to describe the insulating states at $|\nu|=1-3$ observed in experiments. Due to the large moiré supercell, the envelop of a low-energy plane-wave-like basis function defined in Eq. (5) varys slowly across the supercell and can be approximated by a continuous plane- wave function. Then the e-e interaction can be expressed in the plane-wave-like basis as ${ }^{15}$

$$
\begin{aligned}
\hat{H}_{e-e}= & \frac{1}{2 \tilde{N}} \sum_{\sigma \sigma^{\prime}} \sum_{\xi \xi^{\prime}} \sum_{\alpha \beta} \sum_{\mathbf{k k}^{\prime} \mathbf{q}} V_{\alpha \beta}(\mathbf{q}) \\
& c_{\mathbf{k}+\mathbf{q}, \alpha \xi \sigma}^{\dagger} c_{\mathbf{k}^{\prime}-\mathbf{q}, \beta \xi^{\prime} \sigma^{\prime}} c_{\mathbf{k}^{\prime}, \beta \xi^{\prime} \sigma^{\prime}} c_{\mathbf{k}, \alpha \xi \sigma},
\end{aligned}
$$

where $\sigma$ and $\sigma^{\prime}$ are the spin indexes, $V_{\alpha \beta}(\mathbf{q})$ is the ee interaction kernel, $\mathbf{k}=\mathbf{k}^{(s)}+\mathbf{G}^{(s)}$ and $\mathbf{k}^{\prime}=\mathbf{k}^{(s)}+$ $\mathbf{G}^{\prime(s)}$ represent k-points with respect to $\mathbf{k}_{\xi}$ and $\mathbf{k}_{\xi^{\prime}}$, respectively, $\mathbf{k}$ and $\mathbf{k}+\mathbf{q}$ with a small $\mathbf{q}$ are in the same valley and the terms with $\mathbf{k}$ and $\mathbf{k}+\mathbf{q}$ in different valleys are negligible. The $V_{\alpha \beta}(\mathbf{q})$ is given by $e^{2} /\left(\Omega^{(s)} 4 \pi \epsilon_{r} \epsilon_{0}\right) 2 \pi /|\mathbf{q}|$ for sublattices $\alpha$ and $\beta$ in the same layer and $e^{2} /\left(\Omega^{(s)} 4 \pi \epsilon_{r} \epsilon_{0}\right) e^{-|\mathbf{q}| \bar{h}} 2 \pi /|\mathbf{q}|$ for $\alpha$ and $\beta$ in different layers, where $\Omega^{(s)}$ is the supercell area, $\epsilon_{r}$ is the dielectric constant, and $\bar{h}$ is the average interlayer spacing ${ }^{33}$. Here, $\epsilon_{r}$ is taken to be 10 considering the screening by both the BN substrates and electric gates. With this $\epsilon_{r}$, the screened e-e interaction between intralayer nearest neighbors is $1.02 \mathrm{eV}$, which is much smaller than that of the freestanding graphene without dielectric screening from the substrates and electric gates ${ }^{35}$.

We employ the SCHF method similar to that proposed 
(a)
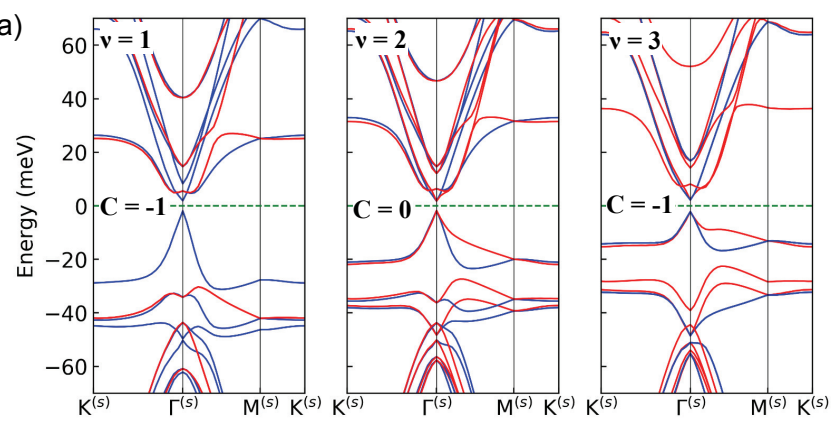

(b)
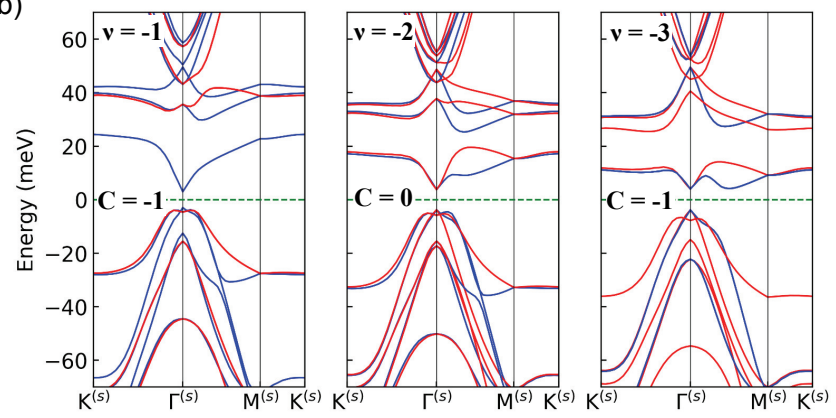

FIG. 5. (Color online) The MF band structures of the relaxed TBG/BN with $\theta=1.08^{\circ}$ and an integer number of filled flat bands in the SCHF ground states. Positive (a) and negative (b) $\nu$ represent the number of filled conduction bands and the number of empty valence bands, respectively. Relative to CNP, one conduction band per valley is filled at $\nu=2$, two (one) conduction bands in the $\xi=+(\xi=-)$ valley are filled at $\nu=3$, one valence band per valley is empty at $\nu=-2$, and two (one) valence bands in the $\xi=+(\xi=-)$ valley are empty at $\nu=-3$. The Fermi levels are set to be zero and are represented by the dashed green lines. The total Chern number $(C)$ of the filled bands is labeled for each $\nu$.

by Xie and MacDonald ${ }^{33}$ to obtain the mean-field (MF) band structures of TBG/BN when the e-e interaction is taken into account. It has been demonstrated that the insulating ground states of the pristine TBG at finite $\nu$ could be properly described by the SCHF method ${ }^{33}$. It is noted that expressing $\hat{H}^{0}$ in the plane-wave-like basis enables us to obtain the SCHF ground states of the fully relaxed TBG/BN, while previous studies only considered rigid TBG or some relaxation effect in TBG with one empirical parameter ${ }^{16,33}$. The MF states with valley- and spin-polarized bands are considered here. In the following, all k-points are for the supercell and the superscript " (s)" in the k-point denotations is omitted for clarity, and $\tau=(\xi, \sigma)$ is used to denote the four flavors of the band states.

Within the SCHF method, the MF Hamiltonian can be written as $\hat{H}_{M F}=\hat{H}^{0}+\hat{H}^{H}+\hat{H}^{F}$. In the plane-wave-like basis, the singleparticle Hamiltonian operator reads $\hat{H}^{0}=$ $1 / N_{\mathbf{k}} \sum_{\sigma \xi} \sum_{\alpha \beta} \sum_{\mathbf{k G G}^{\prime}} H_{\alpha, \mathbf{G}^{\prime} ; \beta, \mathbf{G}}^{0}(\xi, \mathbf{k}) c_{\mathbf{k}+\mathbf{G}^{\prime}, \alpha \xi \sigma}^{\dagger} c_{\mathbf{k}+\mathbf{G}, \beta \xi \sigma}$, where $H_{\alpha, \mathbf{G}^{\prime} ; \beta, \mathbf{G}}^{0}(\xi, \mathbf{k})$ is just the matrix element in Eq. (6). The nonlocal Fock and Hartree operators are given by

$$
\begin{aligned}
\hat{H}^{F}= & -\frac{1}{N_{\mathbf{k}}} \sum_{\tau \mathbf{k}} \sum_{\alpha \beta \mathbf{G} \mathbf{Q}} \sum_{\mathbf{k}^{\prime} \mathbf{G}^{\prime}} V_{\alpha \beta}\left(\mathbf{k}+\mathbf{G}+\mathbf{Q}-\mathbf{k}^{\prime}-\mathbf{G}^{\prime}\right) \\
& \delta \rho_{\beta, \mathbf{G}^{\prime}-\mathbf{Q} ; \alpha, \mathbf{G}^{\prime}}\left(\tau, \mathbf{k}^{\prime}\right) c_{\mathbf{k}+\mathbf{G}+\mathbf{Q}, \alpha \tau}^{\dagger} c_{\mathbf{k}+\mathbf{G}, \beta \tau} \\
\hat{H}^{H}= & \frac{1}{N_{\mathbf{k}}} \sum_{\tau \mathbf{k}} \sum_{\alpha \mathbf{G} \mathbf{Q} \tau^{\prime} \alpha^{\prime} \mathbf{k}^{\prime} \mathbf{G}^{\prime}} V_{\alpha \alpha^{\prime}}(\mathbf{Q}) \\
& \delta \rho_{\alpha^{\prime}, \mathbf{G}^{\prime}-\mathbf{Q} ; \alpha^{\prime}, \mathbf{G}^{\prime}}\left(\tau^{\prime}, \mathbf{k}^{\prime}\right) c_{\mathbf{k}+\mathbf{G}+\mathbf{Q}, \alpha \tau}^{\dagger} c_{\mathbf{k}+\mathbf{G}, \alpha \tau}
\end{aligned}
$$

where $\delta \rho=\rho-\rho_{\text {iso }}$ with $\rho_{\beta, \mathbf{G}^{\prime}-\mathbf{Q} ; \alpha, \mathbf{G}^{\prime}}\left(\tau, \mathbf{k}^{\prime}\right)$ denoting the density matrix element $\left\langle c_{\mathbf{k}^{\prime}+\mathbf{G}^{\prime}-\mathbf{Q}, \beta \tau} c_{\mathbf{k}^{\prime}+\mathbf{G}^{\prime}, \alpha \tau}\right\rangle$ for the MF state and $\rho_{\text {iso }}$ the density matrix for the isolated fixed and rotated graphene layers with band states below the charge neutrality point occupied, and the summation over $\mathbf{k}$ and $\mathbf{k}^{\prime}$ is done in the supercell BZ with a uniform $30 \times 30$ k-point grid. $\rho$ can be calculated as

$\rho_{\beta, \mathbf{G}^{\prime}-\mathbf{Q} ; \alpha, \mathbf{G}^{\prime}}\left(\tau, \mathbf{k}^{\prime}\right)=\sum_{n}^{\text {occupied }} \psi_{\beta, \mathbf{G}^{\prime}-\mathbf{Q}}^{n *}\left(\tau, \mathbf{k}^{\prime}\right) \psi_{\alpha, \mathbf{G}^{\prime}}^{n}\left(\tau, \mathbf{k}^{\prime}\right)$,

where $\psi^{n}\left(\tau, \mathbf{k}^{\prime}\right)$ represents a MF band state with flavor $\tau$ at the k-point $\mathbf{k}^{\prime}$. In Eqs. (8) and (9), 19 shortest reciprocal lattice vectors [see Fig. 7(b)] are used for $\mathbf{Q}$ as the Fock and Hartree elements generally decease with the magnitude of $\mathbf{Q}$. With such a set of $\mathbf{Q}$, the nonlocality of the Fock and Hartree operators is maintained in the calculations. The summation over terms involving $V_{\alpha \beta}(\mathbf{q})$ with $\mathbf{q}$ close to zero in Eq. (8) is done using a similar method in Ref. [36].

The $\hat{H}_{M F}$ can be diagonalized to obtain the MF band states for each $\tau$ and $\mathbf{k}$. We have used the 37 shortest $\mathbf{G}$ shown in Fig. 7(b) for the plane-wave-like basis. Then 148 bands per flavor are updated during the SCHF iterations. The MF Hamiltonian and its total energy are functionals of the density matrix $\rho$. For a filling factor $\nu$, the initial $\rho$ of the self-consistent calculation is taken to be that of the single-particle band states with the filling scheme corresponding to $\nu$. Then a linearly mixed $\rho$ is used for the next iteration. The SCHF iterations are performed until the convergence of the total energy per supercell is reached with a tolerance of $10^{-5} \mathrm{eV}$. The filling scheme is determined by the band-state energies during the iterations, and we find that it remains the same as the initial one for the converged state.

Figure 5 shows the computed MF band structures at $\nu= \pm 1, \pm 2$, and \pm 3 for TBG/BN with $\theta=1.08^{\circ}$. The $\mathrm{SCHF}$ ground states at different $\nu$ are all insulating with narrow MF gaps. The total Chern number $(C)$ of the filled bands is also calculated and are labeled in Fig. 5. At $\nu= \pm 1$ and \pm 3 , a nontrivial $C$ is obtained and the QAH effect may be observed. For $\nu=+1$ and +3 , one more flat band with $C_{n}=-1$ in the $\xi=+$ valley is occupied than the $\xi=-$ valley, and one more flat band with $C_{n}=+1$ in the $\xi=+$ valley is empty than the $\xi=-$ valley for $\nu=-1$ and -3 . 
The MF gaps opened around the $\Gamma^{(s)}$ point are listed in Table I. When the flat conduction bands are filled, the gap at $\nu=1$ is smaller than that at $\nu=3$, the QAH effect is thus more likely to be observed at $\nu=3$. This is roughly consistent with the recent experimental observations $^{12}$. For states with empty valence bands, the gap at $\nu=-3$ is also larger than that $\nu=-1$, while their gaps are larger than those at positive $\nu$. Compared with the experiments ${ }^{12}$, the band gap at $\nu=3$ is overestimated at the MF level, which may be corrected by a higher theoretical level with the dynamically screened ee interaction in future investigations. For TBG/BN with larger $\theta$, the gaps become wider and their values at negative $\nu$ become smaller than those at positive $\nu$, as shown in Table I.

To characterize the nature of the MF band states, the local density of states (LDOS) at the AA-stacked part of G3/G2 is computed for each $\nu$ and is compared with that of the single-particle bands at CNP, as shown in Fig. 6 . The single-particle flat-band states are mainly localized at the AA-stacked part as demonstrated by the two narrow LDOS peaks around the Fermi level $\left(E_{F}\right)$. At finite $\nu$, the bands around $E_{F}$ are still rather flat, while the LDOS peaks are wider than those of the single-particle LDOS. Moreover, three LDOS peaks can be seen. For positive $\nu$, the peak value above $E_{F}$ becomes smaller with more filled bands, while the peak just below $E_{F}$ becomes higher. The LDOS peaks for negative $\nu$ show a similar trend. Such evolution behavior of the LDOS peaks with $\nu$ is roughly consistent with the general trend of the scanning tunnelling spectrum observations on TBG with varying doping levels ${ }^{37}$. In particular, three spectra peaks are observed in experiments away from CNP, while the peak separations are smaller than those for the calculated LDOS at the MF level.

\section{SUMMARY AND CONCLUSIONS}

Full relaxation of the commensurate double moiré superlattices in TBG/BN has been performed. The superlattice in $\mathrm{G} 2 / \mathrm{BN}$ with a twist angle of about $1.6^{\circ}$ becomes commensurate with that in G3/G2 with the magic angle $\theta_{m}$. The inversion-asymmetric spatial variation of the interlayer interaction potential between G2 and BN leads to the broken $C_{2}$ symmetry in the structural deformation in G2, while the symmetry is approximately maintained in G3. For the relaxed TBG/BN, an effective single-particle Hamiltonian $\hat{H}^{0}$ taking into account the relaxation effect and the full effective Hamiltonian introduced by $\mathrm{BN}$ is built for the moiré superlattice in G3/G2. The Hamiltonian terms in $\hat{H}^{0}$ induced by both relaxation and $\mathrm{BN}$ break the $C_{2}$ symmetry, leading to an opened gap at CNP that separates the flat conduction and valence bands. These gapped flat bands have finite Chern numbers. The symmetry breaking in $\hat{H}^{0}$ is found to mainly originate from the intralayer inversionasymmetric strain fields in G2, which induce spatially

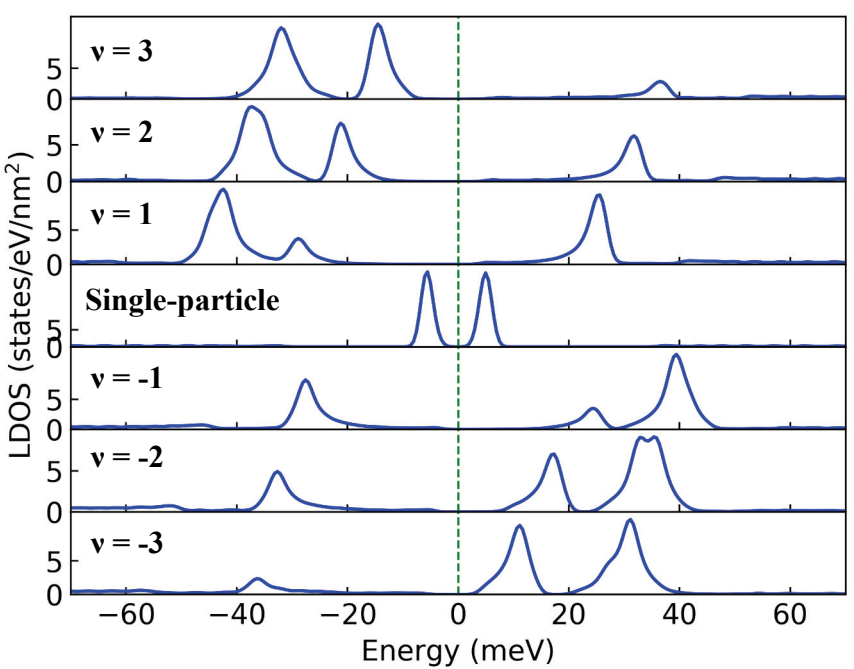

FIG. 6. (Color online) The local density of states (LDOS) at the AA-stacked part of G3/G2 for the MF band states at each finite $\nu$ and the single-particle band states at CNP. The Fermi levels are set to be zero and are represented by the dashed green lines.

non-uniform modifications of the intralayer on-site energies and the nearest-neighbor hopping terms. The influence on the symmetry of the interlayer hopping by the structural deformation in G2 is much weaker than that for the intralayer terms. The MF band structures of the SCHF ground states at different filling factor $\nu$ are acquired based on $\hat{H}^{0}$ in the plane-wave-like basis. For TBG/BN with $\theta_{m}$, the ground states with $|\nu|=1-3$ are all insulating with narrow MF gaps. When the flat conduction bands are filled, the gap at $\nu=1(\nu=-1)$ is smaller than that at $\nu=3(\nu=-3)$. Our study thus suggests that $\mathrm{TBG} / \mathrm{BN}$ is a promising platform for observation of the nontrivial topological properties driven by the broken symmetry.

\section{ACKNOWLEDGMENTS}

We gratefully acknowledge valuable discussions with D. Tománek, D. Liu, H. Xiong, and Q. Zhang. This research was supported by the National Natural Science Foundation of China (Grants No. 11974312 and No. 11774195), and the National Key Research and Development Program of China(Grant No. 2016YFB0700102). The calculations were performed on TianHe-1(A) at National Supercomputer Center in Tianjin. 
(a)

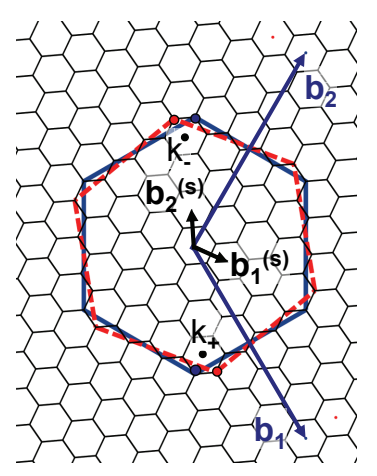

(b)

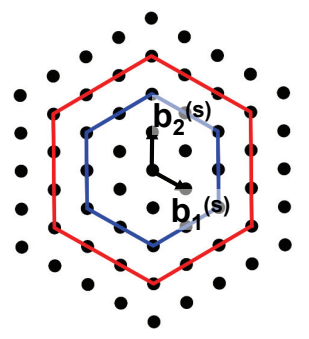

FIG. 7. (Color online). (a) The schematic reciprocal lattice of the moiré superlattice in G3/G2 of TBG/BN. Small hexagons are periodic BZs of the superlattice, spanned by $\mathbf{b}_{\mathbf{1}}^{(\mathbf{s})}$ and $\mathbf{b}_{\mathbf{2}}^{(\mathbf{s})}$. Large hexagons are BZ of the fixed layer (solid), spanned by $\mathbf{b}_{1}$ and $\mathbf{b}_{2}$, and BZ of the twisted layer (dashed). $\mathbf{k}_{\xi}(\xi= \pm$ ) is the center of one of the supercell BZs containing the Dirac points of the fixed and twisted layers at their corners in the valley $\xi$, and we take $\mathbf{k}_{-}=-\mathbf{k}_{+}$. (b) The reciprocal lattice vectors of the superlattice. Inside the red hexagon are the 37 vectors with magnitudes smaller than or equal to $3\left|\mathbf{b}_{\mathbf{1}}^{(\mathbf{s})}\right|$, and the 19 vectors inside the blue hexagon have magnitudes smaller than or equal to $2\left|\mathbf{b}_{\mathbf{1}}^{(\mathbf{s})}\right|$.

\section{APPENDIX}

\section{A. Geometry of the double moiré superlattices in TBG/BN}

We consider the trilayer structures with the top TBG nearly aligned with the bottom BN layer. The top graphene layer (G3) and the bottom BN layer are rotated by $\theta$ and $\theta^{\prime}$ counterclockwise respectively with respect to the fixed middle graphene layer (G2), as shown schematically in Fig. 1(a). Due to the relative twist between the adjacent layers and the lattice-constant mismatch between graphene and $\mathrm{BN}$, double moiré superlattices are formed in TBG/BN, as shown in Fig. 1(b). As directly seen from the moiré patters with small twist angles, the spanning vectors $\left(\mathbf{a}_{\mathbf{j}}^{(\mathbf{s})}\right.$ with $\left.j=1,2\right)$ of the moiré superlattice in G3 on G2 (G3/G2) are along the armchair directions of graphene, while those $\left(\mathbf{a}_{\mathbf{j}}^{(\mathbf{s})^{\prime}}\right)$ of the moiré superlattice in $\mathrm{G} 2$ on $\mathrm{BN}(\mathrm{G} 2 / \mathrm{BN})$ are along the zigzag directions. Here, we study the commensurate double moiré superlattices with small twist angles, then $a^{(s)} \simeq \sqrt{3} a^{(s)^{\prime}}$ for the minimum commensurate supercells, as shown in Fig. 1(b), where $a^{(s)}$ and $a^{(s)^{\prime}}$ are lengthes of $\mathbf{a}_{\mathbf{j}}^{(\mathbf{s})}$ and $\mathbf{a}_{\mathbf{j}}^{(\mathbf{s})^{\prime}}$, respectively. In the following, the geometry parameters of the commensurate superlattices are obtained.

The unit cell of the fixed G2 layer is spanned by the basis vectors $\mathbf{a}_{\mathbf{1}}=a(\sqrt{3} / 2,-1 / 2)^{\mathrm{T}}$ and $\mathbf{a}_{\mathbf{2}}=$ $a(\sqrt{3} / 2,1 / 2)^{\mathrm{T}}$, where the superscript $\mathrm{T}$ denotes matrix transposition. The basis vectors of the BN layer become

$\mathbf{a}_{\mathbf{j}}^{\prime}=S \mathbf{a}_{\mathbf{j}}(j=1,2)$, where the transformation matrix

$$
S=\frac{1}{1+\epsilon}\left(\begin{array}{cc}
\cos \theta^{\prime} & -\sin \theta^{\prime} \\
\sin \theta^{\prime} & \cos \theta^{\prime}
\end{array}\right)
$$

In the G3 layer, the unit cell is spanned by $T_{\theta} \mathbf{a}_{\mathbf{j}}(j=1$, 2 ), where $T_{\theta}$ denotes the counterclockwise rotation by $\theta$.

In G3/G2, the strictly periodic moiré superlattices are spanned by the basis vectors $\mathbf{a}_{\mathbf{1}}^{(\mathbf{s})}=N \mathbf{a}_{\mathbf{1}}+(N+1) \mathbf{a}_{\mathbf{2}}$ and $\mathbf{a}_{\mathbf{2}}^{(\mathbf{s})}=-(N+1) \mathbf{a}_{\mathbf{1}}+(2 N+1) \mathbf{a}_{\mathbf{2}}$, where $N$ is an integer. The relation between $\theta$ and $N$ can be expressed as $\cos \theta=$ $\left(1+6 N+6 N^{2}\right) /\left(2+6 N+6 N^{2}\right)$. For $N=30, \theta$ takes $1.0845^{\circ}$ and is closest to the experimentally observed $\theta_{m}$. The length of $\mathbf{a}_{\mathbf{j}}^{(\mathbf{s})}$ is given by $a^{(s)}=a /[2 \sin (\theta / 2)]$. In G2/BN, the spanning vectors of the moiré superlattices can be taken as $\left(S^{-1}-I\right) \mathbf{a}_{\mathbf{j}}^{(\mathbf{s})}=\mathbf{a}_{\mathbf{j}}$, which gives the moiré period $a^{(s)^{\prime}}=a / \sqrt{\epsilon^{2}+4(1+\epsilon) \sin ^{2}\left(\theta^{\prime} / 2\right)}$. Since $a^{(s)} \simeq$ $\sqrt{3} a^{(s)^{\prime}}$ in the commensurate superlattices, $\theta^{\prime} \simeq 1.6^{\circ}$ for $\theta$ around $\theta_{m}$ and $\epsilon=-1.70 \%$. With the approximate $\theta^{\prime}$, the basis vectors of $\mathrm{BN} \mathbf{a}_{\mathbf{j}}^{\prime}$ can be computed. For $N$ around 30 , we find that $\mathbf{a}_{1}^{(\mathbf{s})}$ can be approximately expressed as $(N+1) \mathbf{a}_{\mathbf{1}}^{\prime}+(N-1) \mathbf{a}_{\mathbf{2}}^{\prime}$, and it can become an exact lattice vector of $\mathrm{BN}$ by varying $\theta^{\prime}$ around $1.6^{\circ}$ and varying $\epsilon$ around $-1.70 \%$ very slightly. Then in the strictly periodic moiré superlattices, $\mathbf{a}_{\mathbf{1}}^{(\mathbf{s})}=N \mathbf{a}_{\mathbf{1}}+(N+$ 1) $\mathbf{a}_{\mathbf{2}}=(N+1) \mathbf{a}_{\mathbf{1}}^{\prime}+(N-1) \mathbf{a}_{\mathbf{2}}^{\prime}$, which gives

$$
\begin{aligned}
\epsilon & =\sqrt{\frac{1+3 N^{2}}{1+3 N+3 N^{2}}}-1, \\
\tan \theta^{\prime} & =\frac{\sqrt{3}(3 N+1)}{6 N^{2}+3 N-1} .
\end{aligned}
$$

The computed $\epsilon$ and $\theta^{\prime}$ for the three considered cases with $N$ from 28 to 30 are listed in Table I. We note that for $N$ outside this range, the deviation of $\epsilon$ from $-1.70 \%$ becomes rather large. In addition, when $\mathrm{BN}$ is rotated clockwise with respect to G2, the deviation of $\epsilon$ from $-1.70 \%$ for the commensurate superlattices is also rather large.

The sublattice-A and sublattice-B atoms in a unit cell of G2 are located at $\left(\mathbf{a}_{\mathbf{1}}+\mathbf{a}_{\mathbf{2}}\right) / 3$ and $\left(2 \mathbf{a}_{\mathbf{1}}+2 \mathbf{a}_{\mathbf{2}}\right) / 3$, respectively. In $\mathrm{BN}$, the lattices formed by the boron and nitrogen atoms are labeled as sublattice-A and sublattice$B$, respectively. For the initial structure without twist and lattice-constant mismatch, the adjacent layers are all taken to be AA-stacked. In TBG/BN, the local stackings between adjacent layers vary continuously and are characterized by the relative shift vectors. At an in-plane position $\mathbf{r}$ in the rigid superlattice, the shift vector between G3 and G2 is taken to be $\boldsymbol{\delta}=\left(I-T_{-\theta}\right) \mathbf{r}$, and the shift vector between $\mathrm{G} 2$ and $\mathrm{BN}$ is given by $\boldsymbol{\delta}^{\prime}=\left(S^{-1}-I\right) \mathbf{r}$. 


\section{B. Relaxation of TBG/BN by Euler-Lagrange equations}

The in-plane structural relaxation in each layer of $\mathrm{TBG} / \mathrm{BN}$ is driven by the interlayer interaction potentials, which vary with the local stacking configurations. At an in-plane position in the superlattice, the local spacings between the adjacent layers are taken to be the optimal values for the local stacking configurations, and the local potentials are taken as the calculated energies of the corresponding bilayers. Between G3 and G2, the interlayer potential $V$ as a function of the local shift vector $\boldsymbol{\delta}$ can be expressed as $V(\boldsymbol{\delta})=\tilde{V} \sum_{j=1}^{3} \cos \left(\mathbf{G}_{j} \cdot \boldsymbol{\delta}\right)$, where the sum is limited to three shortest vectors, $\mathbf{G}_{\mathbf{1}}=\mathbf{b}_{\mathbf{1}}$, $\mathbf{G}_{\mathbf{2}}=\mathbf{b}_{\mathbf{2}}$, and $\mathbf{G}_{\mathbf{3}}=-\mathbf{b}_{\mathbf{1}}-\mathbf{b}_{\mathbf{2}}$, and the parameter obtained from ab-initio calculations is $\tilde{V}=0.817 \mathrm{meV} / \AA^{2}$. The $a b$ - initio computational approach is detailed in Appendix C. It is noted that $V(\boldsymbol{\delta})$ has the inversion symmetry with $V(\boldsymbol{\delta})=V(-\boldsymbol{\delta})$. However, the inversion symmetry is absent in the interlayer potential $V^{\prime}$ between G2 and BN as the two sublattices in BN are composed of different kinds of atoms. The $V^{\prime}$ is thus expressed as $V^{\prime}(\boldsymbol{\delta})=\tilde{V}^{\prime} \sum_{j=1}^{3} \cos \left(\mathbf{G}_{j} \cdot \boldsymbol{\delta}+\phi_{V}^{\prime}\right)$, where $\tilde{V}^{\prime}=0.845$ $\mathrm{meV} / \AA^{2}$ and $\phi_{V}^{\prime}=-50.26^{\circ}$.

We have employed the continuum elastic theory to evaluate $E_{t o t}$ of a supercell. $E_{t o t}$ as the sum of the elastic energy $\left(E_{e l}\right)$ in each layer and the interlayer interaction energy $\left(E_{\text {int }}\right)$ is a functional of the displacement fields $\mathbf{u}^{(\mathbf{n})}(\mathbf{r})$ with $n$ the layer index shown in Fig. 1(a). The elastic energy functional is given by ${ }^{38}$

$$
\begin{gathered}
E_{e l}=\sum_{n=1}^{3} \int d \mathbf{r}\left\{\frac{\lambda_{n}+\mu_{n}}{2}\left(\frac{\partial u_{x}^{(n)}}{\partial x}+\frac{\partial u_{y}^{(n)}}{\partial y}\right)^{2}+\right. \\
\left.\frac{\mu_{n}}{2}\left[\left(\frac{\partial u_{x}^{(n)}}{\partial x}-\frac{\partial u_{y}^{(n)}}{\partial y}\right)^{2}+\left(\frac{\partial u_{y}^{(n)}}{\partial x}+\frac{\partial u_{x}^{(n)}}{\partial y}\right)^{2}\right]\right\}
\end{gathered}
$$

where the integral extends over a moiré supercell. The calculated 2D elastic Lamé factors are $\lambda_{1}=1.779 \mathrm{eV} / \AA^{2}$ and $\mu_{1}=7.939 \mathrm{eV} / \AA^{2}$ for $\mathrm{BN}$ and $\lambda_{2}=\lambda_{3}=$ $3.653 \mathrm{eV} / \AA^{2}$ and $\mu_{2}=\mu_{3}=9.125 \mathrm{eV} / \AA^{2}$ for graphene. The $E_{\text {int }}$ is given by the integral of the local interlayer interaction potentials ${ }^{39}$

$$
E_{i n t}=\int\left\{V[\boldsymbol{\delta}(\mathbf{r})]+V^{\prime}\left[\boldsymbol{\delta}^{\prime}(\mathbf{r})\right]\right\} d \mathbf{r}
$$

where $\boldsymbol{\delta}(\mathbf{r})=\left(I-T_{-\theta}\right) \mathbf{r}+\mathbf{u}^{(3)}(\mathbf{r})-\mathbf{u}^{(2)}(\mathbf{r})$ and $\boldsymbol{\delta}^{\prime}(\mathbf{r})=$ $\left(S^{-1}-I\right) \mathbf{r}+\mathbf{u}^{(2)}(\mathbf{r})-\mathbf{u}^{(1)}(\mathbf{r})$ for the relaxed structure.

The $E_{\text {tot }}$ of a supercell can be expressed as $E_{t o t}=$ $\int L\left[\mathbf{u}^{(1)}, \mathbf{u}^{(2)}, \mathbf{u}^{(3)}\right] d \mathbf{r}$. The minimization of $E_{t o t}$ as a functional of $\mathbf{u}^{(n)}$ leads to a series of Euler-Lagrange equations $^{22}$

$$
\frac{\partial}{\partial x}\left[\frac{\partial L}{\partial\left(\partial u_{\nu}^{(n)} / \partial x\right)}\right]+\frac{\partial}{\partial y}\left[\frac{\partial L}{\partial\left(\partial u_{\nu}^{(n)} / \partial y\right)}\right]-\frac{\partial L}{\partial u_{\nu}^{(n)}}=0
$$

where $\nu=x, y$. To solve these equations, $\mathbf{u}^{(\mathbf{n})}(\mathbf{r})$ is expanded in Fourier series as

$$
\mathbf{u}^{(\mathbf{n})}(\mathbf{r})=\sum_{\mathbf{G}^{(\mathbf{s})}} \tilde{\mathbf{u}}^{(\mathbf{n})}\left(\mathbf{G}^{(\mathbf{s})}\right) \mathbf{e}^{\mathbf{i G}^{(\mathbf{s})} \cdot \mathbf{r}}
$$

where the summation is over nonzero reciprocal lattice vectors $\mathbf{G}^{(\mathbf{s})}$ of the supercell. The $\partial V / \partial \boldsymbol{\delta}$ and $\partial V^{\prime} / \partial \boldsymbol{\delta}^{\prime}$ are also expanded as $\sum_{\mathbf{G}^{(\mathbf{s})}} \tilde{\mathbf{f}}\left(\mathbf{G}^{(\mathbf{s})}\right) e^{i \mathbf{G}^{(\mathbf{s})} \cdot \mathbf{r}}$ and $\sum_{\mathbf{G}^{(\mathbf{s})}} \tilde{\mathbf{f}}^{\prime}\left(\mathbf{G}^{(\mathbf{s})}\right) e^{i \mathbf{G}^{(\mathbf{s})} \cdot \mathbf{r}}$, respectively. Substitution of these Fourier expansions into Eq. (A6) leads to

$$
\begin{array}{cc}
-\left(\begin{array}{cc}
\left(\lambda_{n}+2 \mu_{n}\right) q_{x}^{2}+\mu_{n} q_{y}^{2} & \left(\lambda_{n}+\mu_{n}\right) q_{x} q_{y} \\
\left(\lambda_{n}+\mu_{n}\right) q_{x} q_{y} & \left(\lambda_{n}+2 \mu_{n}\right) q_{y}^{2}+\mu_{n} q_{x}^{2}
\end{array}\right) & \left(\begin{array}{c}
u_{x}^{(n)}(\mathbf{q}) \\
u_{y}^{(n)}(\mathbf{q})
\end{array}\right) \\
=\tilde{F}_{n}(\mathbf{q}), & (\mathrm{A} 8)
\end{array}
$$

where $\mathbf{q}$ takes each $\mathbf{G}^{(\mathbf{s})}, \tilde{F}_{1}=\left(-\tilde{f}_{x}^{\prime},-\tilde{f}_{y}^{\prime}\right)^{\mathrm{T}}, \tilde{F}_{2}=\left(\tilde{f}_{x}^{\prime}-\right.$ $\left.\tilde{f}_{x}, \tilde{f}_{y}^{\prime}-\tilde{f}_{y}\right)^{\mathrm{T}}$, and $\tilde{F}_{3}=\left(\tilde{f}_{x}, \tilde{f}_{y}\right)^{\mathrm{T}}$.

\section{Ab-initio computational approach}

The ab-initio density functional theory (DFT) calculations of the shifted bilayers are performed using the VASP code ${ }^{40,41}$ to extract the required model parameters. The local density approximation (LDA $)^{42}$ functional is adopted. No van der Waals (vdW) functional has been used, while the vdW interaction is partly accounted by the LDA functional ${ }^{43}$. The projector augmented wave (PAW) potentials ${ }^{44,45}$ are used with a kinetic energy cutoff of $600 \mathrm{eV}$. The BZ sampling is done using a $36 \times 36 \times 1$ Monkhorst-Pack $(\mathrm{MP})$ grid $^{46}$. The vacuums in the $z$ direction are larger than $17 \AA$. The tolerance for the energy convergence is $10^{-6} \mathrm{eV}$. The optimal interlayer distances $(h)$ of the shifted bilayers are obtained by minimizing the total energies through adaptively scanning $h$ with a final precision of $0.001 \AA$.
* E-mail: xqlin@zjut.edu.cn

1 Y. Cao, V. Fatemi, A. Demir, S. Fang, S. L. Tomarken, J. Y. Luo, J. D. Sanchez-Yamagishi, K. Watanabe, T. Taniguchi, E. Kaxiras, R. C. Ashoori, and P. JarilloHerrero, "Correlated insulator behaviour at half-filling in magic-angle graphene superlattices," Nature (London)
556, 80 (2018).

${ }^{2}$ Y. Cao, V. Fatemi, S. Fang, K. Watanabe, T. Taniguchi, E. Kaxiras, and P. Jarillo-Herrero, "Unconventional superconductivity in magic-angle graphene superlattices," Nature (London) 556, 43 (2018). 
3 A. L. Sharpe, E. J. Fox, A. W. Barnard, J. Finney, K. Watanabe, T. Taniguchi, M. A. Kastner, and D. Goldhaber-Gordon, "Emergent ferromagnetism near three-quarters filling in twisted bilayer graphene," Science 365, 605 (2019).

${ }^{4}$ X. Lu, P. Stepanov, W. Yang, M. Xie, M. A. Aamir, I. Das, C. Urgell, K. Watanabe, T. Taniguchi, G. Zhang, A. Bachtold, A. H. MacDonald, and D. K. Efetov, "Superconductors, orbital magnets, and correlated states in magic angle bilayer graphene," Nature (London) 574, 653 (2019).

5 A. Uri, S. Grover, Y. Cao, J.A. Crosse, K. Bagani, D. Rodan-Legrain, Y. Myasoedov, K. Watanabe, T. Taniguchi, P. Moon, M. Koshino, P. Jarillo-Herrero, and E. Zeldov, "Mapping the twist-angle disorder and Landau levels in magic-angle graphene," Nature 581, 47 (2020).

6 R. Bistritzer and A. H. MacDonald, "Moiré bands in twisted double-layer graphene," Proc. Natl. Acad. Sci. U.S.A. 108, 12233 (2011).

7 G. Trambly de Laissardière, D. Mayou, and L. Magaud, "Numerical studies of confined states in rotated bilayers of graphene," Phys. Rev. B 86, 125413 (2012).

8 J. M. B. Lopes dos Santos, N. M. R. Peres, and A. H. Castro Neto, "Continuum model of the twisted graphene bilayer," Phys. Rev. B 86, 155449 (2012).

9 S. Fang and E. Kaxiras, "Electronic structure theory of weakly interacting bilayers," Phys. Rev. B 93, 235153 (2016).

10 X. Lin and D. Tománek, "Minimum model for the electronic structure of twisted bilayer graphene and related structures," Phys. Rev. B 98, 081410 (2018).

11 G. Tarnopolsky, A. Jura Kruchkov, and A. Vishwanath, "Origin of Magic Angles in Twisted Bilayer Graphene," Phys. Rev. Lett. 122, 106405 (2019).

12 M. Serlin, C. L. Tschirhart, H. Polshyn, Y. Zhang, J. Zhu, K. Watanabe, T. Taniguchi, L. Balents, and A. F. Young, "Intrinsic quantized anomalous Hall effect in a moiré heterostructure," Science 367, 900 (2020).

13 N. Bultinck, S. Chatterjee, and M. P. Zaletel, "Mechanism for Anomalous Hall Ferromagnetism in Twisted Bilayer Graphene," Phys. Rev. Lett. 124, 166601 (2020).

14 Y.-H. Zhang, D. Mao, and T. Senthil, "Twisted bilayer graphene aligned with hexagonal boron nitride: Anomalous Hall effect and a lattice model," Phys. Rev. Research 1, 033126 (2019).

15 J. Liu and X. Dai, "Spontaneous symmetry breaking and topology in twisted bilayer graphene: the nature of the correlated insulating states and the quantum anomalous Hall effect," arXiv:1911.03760 .

16 Y. Zhang, K. Jiang, Z. Wang, and F. Zhang, "Correlated insulating phases of twisted bilayer graphene at commensurate filling fractions: a Hatree-Fock study," arXiv:2001.02476 .

17 J. S. Alden, A. W. Tsen, P. Y. Huang, R. Hovden, L. Brown, J. Park, D. A. Muller, and P. L. McEuen, "Strain solitons and topological defects in bilayer graphene," Proc. Natl. Acad. Sci. U.S.A. 110, 11256 (2013).

18 K. Uchida, S. Furuya, J.-I. Iwata, and A. Oshiyama, "Atomic corrugation and electron localization due to Moiré patterns in twisted bilayer graphenes," Phys. Rev. B 90, 155451 (2014).

19 M. M. van Wijk, A. Schuring, M. I. Katsnelson, and A. Fasolino, "Relaxation of Moiré patterns for slightly misaligned identical lattices: graphene on graphite," 2D Mater. 2, 034010 (2015).

20 S. Dai, Y. Xiang, and D. J. Srolovitz, "Twisted bilayer graphene: Moiré with a twist," Nano Lett. 16, 5923 (2016).

21 S. K. Jain, V. Juričić, and G. T. Barkema, "Structure of twisted and buckled bilayer graphene," 2D Mater. 4, 015018 (2017).

22 N. N. T. Nam and M. Koshino, "Lattice relaxation and energy band modulation in twisted bilayer graphene," Phys. Rev. B 96, 075311 (2017).

23 F. Gargiulo and O. V. Yazyev, "Structural and electronic transformation in low-angle twisted bilayer graphene," $2 \mathrm{D}$ Mater. 5, 015019 (2018).

24 S. Carr, D. Massatt, S. B. Torrisi, P. Cazeaux, M. Luskin, and E. Kaxiras, "Relaxation and domain formation in incommensurate two-dimensional heterostructures," Phys. Rev. B 98, 224102 (2018).

${ }^{25}$ X. Lin, D. Liu, and D. Tománek, "Shear instability in twisted bilayer graphene," Phys. Rev. B 98, 195432 (2018).

${ }^{26}$ H. Yoo, R. Engelke, S. Carr, S. Fang, K. Zhang, P. Cazeaux, S. H. Sung, R. Hovden, A. W. Tsen, T. Taniguchi, K. Watanabe, G.-C. Yi, M. Kim, M. Luskin, E. B. Tadmor, E. Kaxiras, and P. Kim, "Atomic and electronic reconstruction at the van der waals interface in twisted bilayer graphene," Nat. Mater. 18, 448 (2019).

27 P. Lucignano, D. Alfè, V. Cataudella, D. Ninno, and G. Cantele, "Crucial role of atomic corrugation on the flat bands and energy gaps of twisted bilayer graphene at the magic angle $\theta \sim 1.08^{\circ}$," Phys. Rev. B 99, 195419 (2019).

28 F. Guinea and N. R. Walet, "Continuum models for twisted bilayer graphene: Effect of lattice deformation and hopping parameters," Phys. Rev. B 99, 205134 (2019).

29 G. J. Slotman, M. M. van Wijk, P.-L. Zhao, A. Fasolino, M. I. Katsnelson, and S. J. Yuan, "Effect of Structural Relaxation on the Electronic Structure of Graphene on Hexagonal Boron Nitride," Phys. Rev. Lett. 115, 186801 (2015).

30 J. Jung, A. M. DaSilva, A. H. MacDonald, and S. Adam, "Origin of band gaps in graphene on hexagonal boron nitride," Nat. Commun. 6, 6308 (2015).

31 J. Jung, E. Laksono, A. M. DaSilva, A. H. MacDonald, M. Mucha-Kruczyński, and S. Adam, "Moiré band model and band gaps of graphene on hexagonal boron nitride," Phys. Rev. B 96, 085442 (2017).

32 X. Lin and J. Ni, "Effective lattice model of graphene moiré superlattices on hexagonal boron nitride," Phys. Rev. B 100, 195413 (2019).

33 M. Xie and A. H. MacDonald, "Nature of the Correlated Insulator States in Twisted Bilayer Graphene," Phys. Rev. Lett. 124, 097601 (2020).

34 X. Lin, H. Zhu, and J. Ni, "Pressure-induced gap modulation and topological transitions in twisted bilayer and twisted double bilayer graphene," Phys. Rev. B 101, 155405 (2020).

35 T. O. Wehling, E. Şaşıŏ̆lu, C. Friedrich, A. I. Lichtenstein, M. I. Katsnelson, and S. Blügel, "Strength of effective coulomb interactions in graphene and graphite," Phys. Rev. Lett. 106, 236805 (2011).

${ }^{36}$ F. Gygi and A. Baldereschi, "Self-consistent Hartree-Fock and screened-exchange calculations in solids: Application to silicon," Phys. Rev. B 34, 4405 (1986).

37 Y. Xie, B. Lian, B. Jäck, X. Liu, C.-L. Chiu, K. Watanabe, T. Taniguchi, B. A. Bernevig, and A. Yazdani, "Spectroscopic signatures of many-body correlations in magic-angle 
twisted bilayer graphene," Nature 572, 101 (2019).

38 P. L. de Andres, F. Guinea, and M. I. Katsnelson, "Bending modes, anharmonic effects, and thermal expansion coefficient in single-layer and multilayer graphene," Phys. Rev. B 86, 144103 (2012).

39 S. Zhou, J. Han, S. Dai, J. Sun, and D. J. Srolovitz, "Van der Waals bilayer energetics: Generalized stacking-fault energy of graphene, boron nitride, and graphene/boron nitride bilayers," Phys. Rev. B 92, 155438 (2015).

40 G. Kresse and J. Fürthmuller, "Efficiency of ab-initio total energy calculations for metals and semiconductors using a plane-wave basis set," Comp. Mater. Sci. 6, 15 (1996).

41 G. Kresse and J. Fürthmuller, "Efficient iterative schemes for ab initio total-energy calculations using a plane-wave basis set," Phys. Rev. B 54, 11169 (1996).

42 J. P. Perdew and A. Zunger, "Self-interaction correction to density-functional approximations for many-electron systems," Phys. Rev. B 23, 5048 (1981).

43 J. Jung, A. Raoux, Z. Qiao, and A. H. MacDonald, "Ab initio theory of Moiré superlattice bands in layered twodimensional materials," Phys. Rev. B 89, 205414 (2014).

44 P. E. Blöchl, "Projector augmented-wave method," Phys. Rev. B 50, 17953 (1994).

45 G. Kresse and D. Joubert, "From ultrasoft pseudopotentials to the projector augmented-wave method," Phys. Rev. B 59, 1758 (1999).

46 H. J. Monkhorst and J. D. Pack, "Special points for Brillouin-zone integrations," Phys. Rev. B 13, 5188 (1976). 